\title{
Mobile phone technology in chronic disease management
}

Holly Blake, School of Nursing, Faculty of Medicine and Health Sciences, University of Nottingham, Nottingham. Email: Holly.Blake@nottingham.ac.uk

Citation: Blake H (2008) Mobile phone technology in chronic disease management. Nursing Standard. 23, 12, 43-46. Date of acceptance: July 92008.

\begin{abstract}
Summary
Mobile phones are being used to improve nurse-patient communication and monitor health outcomes in chronic disease. Innovative applications of mobile technology are expected to increase over time in community management of cancer, heart disease, asthma and diabetes. This article focuses on mobile phone technology and its contribution to health care.
\end{abstract}

\section{Introduction}

The capabilities and acessibility of new technologies have been recognised in preventing, diagnosing, monitoring and treating disease, and in the development and delivery of preventive measures and proactive health promotion programmes. These technologies include internet, email and mobile phone applications, and are often referred to in health care as 'electronic health' or 'eHealth' (Pagliari et al 2005). While eHealth incorporates a range of electronic communication technologies, this article focuses on the application of mobile phones for capturing nursing documentation, monitoring health values and collecting data to demonstrate health promotion outcomes in patient care and research.

\section{Applications of mobile phones}

Mobile phones are an integral part of everyday life, although mobile phone technology is a relatively new and innovative eHealth methodology. A significant proportion of the UK population owns or has access to a mobile phone, and this form of technology is generally well accepted by most age groups (Office for National Statistics (ONS) 2007). Some nurses and medical staff use mobile phones during home visits to improve communication with hospital or community services.

Health informatics and information technology in nursing practice are increasingly recognised in government policy, such as the Department of Health's Connecting for Health Programme, which delivers the National Programme for IT (NPfIT) (House of Commons Committee of Public Accounts 2007). Text messages are used in the NHS in a combined email and text directory service called 'contact', approved for the transmission of patient data. Short message service (SMS) texts can be used to maximise the efficiency, effectiveness and equity of health delivery through improved health communication.

Mobile phone technology is used in a wide variety of settings although few research studies have formally evaluated its impact in monitoring chronic disease. An overview of the application of mobile phones for wireless monitoring of health outcomes and the delivery of health promotion interventions has been presented 
(Blake 2008a). There is emerging literature on the application of mobile phones in healthcare delivery, although published evidence is limited. As technology continues to develop, mobile phones will become increasingly important in the strategic implementation of health monitoring schemes.It is therefore important that nurses are aware of innovations in this field.

Using mobile technology in health promotion programmes has advanced their delivery by providing personalised messaging to participants and efficient data collection. Research evidence suggests that mobile technology may be an option for intervention in diet or weight management (Kubota et al 2004, Wang et al 2006), physical activity (Consolvo et al 2006, Hurling et al 2007), smoking (Lazev et al 2004, Obermayer et al 2004, Bramley et al 2005, Rodgers et al 2005, Vidrine et al 2006a, 2006b), and drug and alcohol consumption (Collins et al 2003, Wilkins et al 2003, Freedman et al 2006). Patients are offered mobile phone services that use the SMS text messaging or voice response function to help them manage their disease.This can include: accessing test results; self-monitoring of health and providing objective data to nursing and medical teams, such as their blood pressure, cardiac pulsation and electrocardiogram, weight and step counts; and subjective data, such as self-reported quality of life questionnaires.

There are few published studies that focus on mobile phone technology for disease management and health monitoring. However, the technology is highly topical and the literature reports rapid advancement in the field, for example in the management of cancer (Bielli et al 2004, Maguire et al 2008), asthma (Anhøj and Møldrup 2004), diabetes (Giménez-Pérez et al 2002, Farmer et al 2005, Gammon et al 2005, Carroll et al 2007, Kim and Jeong 2007), and the care of older people (Miskelly 2005). A review identified that studies have been conducted predominantly in Europe (Italy, Denmark, Norway, Spain and the UK), Korea and the United States (US) (Blake 2008b). These are largely descriptive with few controlled trials.

\section{Monitoring health status}

It is often difficult for nursing and medical staff to obtain information about patients' health status once they have returned home. In Italy Bielli et al (2004) developed a wireless health outcomes monitoring system (WHOMS) to improve communication between medical teams and patients about symptoms and quality of life. The WHOMS system allowed structured questionnaires to be sent directly to a patient's mobile phone by the medical team. Patients could use the phone keypad to transfer their responses automatically to an authorised website. This information was then accessed by the medical team and provided graphical representation of the patient's health status.

Bielli et al (2004) tested the WHOMS with 97 cancer inpatients and found that more than half successfully completed a health-related quality of life questionnaire with minimal missing data. However, $42 \%$ of the patients refused to use the system, which suggests that compliance could be an issue, particularly for those individuals who may be less familiar with the technology. This has implications for health education.

Bielli et al (2004) suggest that using mobile technology might be feasible for accessing cancer patients in the community. The proportion of users might also increase in practice as patients who are not familiar with use of the technology may be 
able to access help from family or friends to transfer data. The study highlights some issues with acceptability of technology, particularly for older individuals who may be less familiar with mobile phones in everyday life (Bielli et al 2004). However, data suggest that older adults are more likely to use mobile phones than the internet (ONS 2007), and, as technology infiltrates more aspects of daily living, generational differences in use are likely to decline.

A randomised controlled trial reported on the perceptions of nurses $(n=35)$ towards a mobile phone-based system (Advanced Symptom Management System (ASyMS ${ }^{\odot}$ ) in managing chemotherapy-related toxicity in patients with breast, lung and colorectal cancer (Maguire et al 2008). Many patients receive chemotherapy as outpatients without direct support from oncology professionals. Information and communication technology can be used to support these patients at home. The nurses' overall perceptions of this system were positive and they recognised the benefits of using mobile phones in early intervention and in the management of symptoms.

Although the use of mobile phones in health care is increasing, they are not extensively used as many practitioners may still favour more traditional methods of monitoring and data collection. Advances in mobile phone functionality and application therefore need to be matched by advances in nursing education, with identification and discussion of unresolved issues in practice.

\section{Electronic tracking}

In the UK, Miskelly (2005) reported the use of mobile phone alerts for the electronic tracking of patients with dementia and wandering. Findings were promising and showed that it was possible to locate patients with accuracy and reliability using a global positioning system (GPS) enabled mobile phone. However, this system failed when the relative or carer did not understand how to set up the phone correctly and it showed that there could be training issues related to user compliance. The use of electronic tracking in this way is controversial (O'Neill 2003). While it may provide older people with increased independence, and provide peace of mind for carers, it may also restrict privacy and imply that technology is a substitute for genuine care. Although there is still a paucity of published research in this field, this work highlights that there is potential for mobile phone tracking systems to supplement patient care.

\section{Self-management}

The internet has been proposed as a tool to assist in the self-management of asthma, although web-based asthma diaries, like any web-based programme, are often susceptible to high rates of attrition. Asthma diary data can be collected efficiently by mobile phone. In a small Danish study, Anhøj and Møldrup (2004) used SMS to send 12 patients four text messages each day and encouraged them to respond to at least three on a daily basis. The messages reminded patients to take medication and requested peak flow, sleep loss and medication dosage data. The system was used during the two-month study period and did not decrease over time, as is often observed with web-based tools. More than half of the patients reported two thirds of requested data, although in subsequent focus groups patients suggested that a lower number of daily messages and graphical display of results could improve the system (Anhøj and Møldrup 2004). The system appeared efficient for many patients but the 
outcomes need to be tested in a controlled trial with a larger sample.

Mobile phone technology has most often been applied to the distance monitoring of blood glucose in child and adult diabetes. In interviews with 244 adults with type 1 diabetes in Spain, Giménez-Pérez et al (2002) found that while there was a low rate of internet use for health-related purposes, three quarters of the sample owned a mobile phone, with almost all patients using them more than once a week. The authors concluded that mobile phones therefore have potential for managing diabetes in the community.

Anhøj and Møldrup's (2004) work with asthma patients suggests that graphics may improve mobile phone monitoring systems. Farmer et al (2005) described the development and implementation of a real-time telemedicine system, which was able to transmit blood glucose data from a blood glucose monitor using a mobile phone and collect data on physical activity levels, eating patterns and insulin dosage. In this UK study patients received detailed feedback on their phones including colourful histograms charting glycaemic control over the previous two weeks. This information was accessible to medical staff who could monitor patients' blood glucose levels, identify individuals who were not testing and access charts showing insulin dose patterns and how they can be modified by diet and exercise behaviours (Farmer et al 2005). This system has been formally tested in primary and secondary care environments, which suggests it has potential for wide-scale implementation.

Patients with type 2 diabetes have been monitored using mobile phone technology in nurse-led services. In a controlled study, intervention participants $(n=25)$ were requested by nurses to enter into a website their blood glucose level, and diet and exercise behaviours each day using a mobile phone or wireless internet source (Kim and Jeong 2007). Mobile phone text messages were used weekly by a nurse researcher to send optimal recommendations to each patient over a six-month period. Control participants received usual care without the mobile phone intervention. Results were promising as glycosylated haemoglobin $\left(\mathrm{HbA}_{1 \mathrm{c}}\right)$ decreased $1.15 \%$ points at three months and $1.05 \%$ points at six months compared with baseline in the intervention group. Patients in the intervention group had a decrease of two hours post-meal glucose of $85.1 \mathrm{mg} / \mathrm{dl}$ at three months and $63.1 \mathrm{mg} / \mathrm{dl}$ at six months compared with baseline (Kim and Jeong 2007). The study shows the potential of nurse-led mobile phone messaging services in managing patients with diabetes, although the sample was small and more work needs to be done to assess compliance in the long term.

Improving health communication Mobile telecommunication systems have been tested with children who have type 1 diabetes. As well as improving communication between patients and healthcare professionals, there is potential for this form of technology to improve communication between parents and their children. A Norwegian study (Gammon et al 2005) reported the findings of a mobile and wireless system, which was used in the self-management of children with type 1 diabetes to improve communication with parents. The system automatically transferred readings from the child's blood glucose monitor directly to the parent's mobile phone. Parent and child experiences were evaluated using questionnaires and interviews.

Given the acceptability of mobile phones as part of everyday life, the system was 
effective and well received when the child regularly measured his or her blood glucose level, as it was perceived as a method by which parents could maintain control of monitoring while allowing their child some independence. Difficulties were observed when the child was irregular in his or her self-monitoring, as this resulted in parents having to increase their reminders, which could be negatively viewed by the child (Gammon et al 2005).

Parents had concerns about the appropriateness of the system for adolescents (Gammon et al 2005). However, work in the US by Carroll et al (2007) demonstrated that mobile phone interventions can be effective in adolescents with diabetes. Carroll et al's (2007) prototype diabetes monitoring system integrated a blood glucose monitoring device into the battery pack of a mobile phone. The small-scale evaluation showed that the device had potential for transmitting self-monitoring data directly from the phone to a website where it could be reviewed by medical teams, parents and patients. Furthermore, the adolescents found the system useful and easy to use (Carroll et al 2007).

\section{Discussion}

The benefits of mobile phones to assist nurses with the distance monitoring of patients with chronic disease are evident. In practice mobile technology is beginning to play an important role in improving the provision and transfer of patient records, and in enhancing appointment booking systems by SMS text message reminders to reduce the rates of patients failing to attend appointments, and to inform practitioners of cancelled or rearranged appointments. Mobile phone technology enables practitioners to observe patients' vital signs and activity levels, track health status and detect problems by identifying early warning signs.

Mobile phones can be used to collect small amounts of data frequently and efficiently, and require no manual data entry on receipt, therefore reducing the possibility of record errors. It is clear that there is a consumer market for such systems and further work is under way to enhance the interface and improve accessibility and choice, including the integration of mobile phones with palm computers, speech recognition and interactive voice responders to provide more individual choice in methods for response.

\section{Conclusion}

While mobile phones are widely accessible and appear to be an efficient method of health communication, opinions are mixed on the success, cost benefit and practicality of such interventions. More research is needed to assess the impact of education programmes in eHealth and to evaluate the clinical effectiveness and cost effectiveness of nurse-led mobile phone interventions

\section{References}

Anhøj J, Møldrup C (2004) Feasibility of collecting diary data from asthma patients through mobile phones and SMS (short message service): response rate analysis and focus group evaluation from a pilot study. Journal of Medical Internet Research. 6, 4, 
e42.

Bielli E, Carminati F, La Capra S, Lina M, Brunelli C, Tamburini M (2004) A wireless Health Outcomes Monitoring System (WHOMS): development and field testing with cancer patients using mobile phones. BMC Medical Informatics and Decision Making. June 15, 4, 7.

Blake H (2008a) Innovation in practice: mobile phone technology in patient care. British Journal of Community Nursing. 13, 4, 160, 162-165.

Blake H (2008b) Using technology in health promotion interventions. In Blakely MR, Timmons SM (Eds) Life Style and Health Research. Nova Publishers, New York NY, 17-76.

Bramley D, Riddell T, Whittaker R et al (2005) Smoking cessation using mobile phone text messaging is as effective in Maori as non-Maori. New Zealand Medical Journal. 118, 1216, U1494.

Carroll AE, Marrero DG, Downs SM (2007) The HealthPia GlucoPackTM Diabetes phone: a usability study. Diabetes Technology and Therapeutics. 9, 2, 158-164.

Collins RL, Kashdan TB, Gollnisch G (2003) The feasibility of using cellular phones to collect ecological momentary assessment data: application to alcohol consumption. Experimental and Clinical Psychopharmacology. 11, 1, 73-78.

Consolvo S, Everitt K, Smith I, Landay JA (2006) Design Requirements for Technologies that Encourage Physical Activity. Conference on Human Factors in Computing Systems (April 22-27), Montréal QC, Canada.

Farmer A, Gibson O, Hayton P et al (2005) A real-time, mobile phone-based telemedicine system to support young adults with type 1 diabetes. Informatics in Primary Care. 13, 3, 171-177.

Freedman MJ, Lester KM, McNamara C, Milby JB, Schumacher JE (2006) Cell phones for ecological momentary assessment with cocaine-addicted homeless patients in treatment. Journal of Substance Abuse Treatment. 30, 2, 105-111.

Gammon D, Arsand E, Walseth OA, Andersson N, Jenssen M, Taylor T (2005) Parent-child interaction using a mobile and wireless system for blood glucose monitoring. Journal of Medical Internet Research. 7, 5, e57.

Giménez-Pérez G, Gallach M, Acera E et al (2002) Evaluation of accessibility and use of new communication technologies in patients with type 1 diabetes mellitus. Journal of Medical Internet Research. 4, 3, e16.

House of Commons Committee of Public Accounts (2007) Department of Health: The National Programme for IT in the NHS. Twentieth report of Session 2006/07. March 26. The Stationery Office, London.

Hurling R, Catt M, Boni MD et al (2007) Using internet and mobile phone technology to deliver an automated physical activity program: randomized controlled trial. Journal of Medical Internet Research. 9, 2, e7. 
Kim HS, Jeong HS (2007) A nurse short message service by cellular phone in type-2 diabetic patients for six months. Journal of Clinical Nursing. 16, 6, 1082-1087.

Kubota A, Fujita M, Hatano Y (2004) Development and effects of a health promotion program utilizing the mail function of mobile phones. Nippon Koshu Eisei Zasshi. 51, $10,862-873$.

Lazev AB, Vidrine DJ, Arduino RC, Gritz ER (2004) Increasing access to smoking cessation treatment in a low-income, HIV-positive population: the feasibility of using cellular telephones. Nicotine and Tobacco Research. 6, 2, 281-286.

Maguire R, McCann L, Miller M, Kearney N (2008) Nurse's perceptions and experiences of using of a mobile-phone-based Advanced Symptom Management System (ASyMSC) to monitor and manage chemotherapy-related toxicity. European Journal of Oncology Nursing. 12, 4, 380-386.

Miskelly F (2005) Electronic tracking of patients with dementia and wandering using mobile phone technology. Age and Ageing. 34, 5, 497-499.

Obermayer JL, Riley WT, Asif O, Jean-Mary J (2004) College smoking-cessation using cell phone text messaging. Journal of American College Health. 53, 2, 71-78.

Office for National Statistics (2007) Focus on the Digital Age. www.statistics.gov.uk/focuson/ digitalage/ (Last accessed: November 5 2008.)

O'Neill DJ (2003) Electronic tagging of people with dementia. Tagging should be reserved for babies, convicted criminals, and animals. British Medical Journal. 326, $7383,281$.

Pagliari C, Sloan D, Gregor P et al (2005) What is eHealth (4): a scoping exercise to map the field. Journal of Medical Internet Research. 7, 1, e9.

Rodgers A, Corbett T, Bramley D et al (2005) Do u smoke after txt? Results of a randomised trial of smoking cessation using mobile phone text messaging. Tobacco Control. 14, 4, 255-261.

Vidrine DJ, Arduino RC, Gritz ER (2006a) Impact of a cell phone intervention on mediating mechanisms of smoking cessation in individuals living with HIV/AIDS. Nicotine and Tobacco Research. 8, Suppl 1, S103-S108.

Vidrine DJ, Arduino RC, Lazev AB, Gritz ER (2006b) A randomized trial of a proactive cellular telephone intervention for smokers living with HIV/AIDS. AIDS. $20,2,253-260$.

Wang DH, Kogashiwa M, Kira S (2006) Development of a new instrument for evaluating individuals' dietary intakes. Journal of the American Dietetic Association. $106,10,1588-1593$.

Wilkins C, Casswell S, Barnes H, Pledger M (2003) A pilot study of a computerassisted cell-phone interview (CACI) methodology to survey respondents in households without telephones about alcohol use. Drug and Alcohol Review. 22, 2, 221-225. 